\title{
Hemodialysis vascular access in children - A retrospective study in a pediatric dialysis unit
}

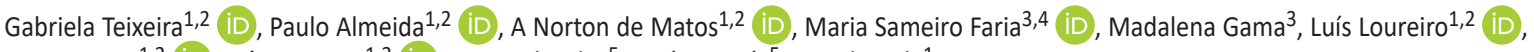 \\ Duarte Rego $^{1,2}$ iD, Inês Antunes ${ }^{1,2}$ iD, Fernanda Silva ${ }^{5}$, José Queirós ${ }^{5}$, Rui Almeida ${ }^{1}$ \\ ${ }^{1}$ Angiology and Vascular Surgery Department, Hospital Santo António - Centro Hospitalar Universitário do Porto, Porto - Portugal \\ ${ }^{2}$ Grupo de Estudos Vasculares, GEV, Porto - Portugal \\ 3 Pediatric Department, Nephrology Unit, Centro Materno-Infantil do Norte - Centro Hospitalar Universitário do Porto, Porto - Portugal \\ ${ }^{4}$ Research Unit on Applied Molecular Biosciences, UCIBIO\REQUIMTE \\ ${ }^{5}$ Nephrology Department, Hospital Santo António - Centro Hospitalar Universitário do Porto, Porto - Portugal
}

\section{ABSTRACT}

Objective: Permanent access in the form of a fistula is the preferred form of vascular access for most pediatric patients on maintenance hemodialysis (HD) therapy; however, the technical aspects of the procedure that are unique to the pediatric population, the expectation of a short waiting time for kidney transplantation and the need to cannulate every other day (with the pain and fear associated with it) limit its use. Our objective was to analyze the long-term outcomes of pediatric arteriovenous fistulas in our institution.

Methods: A retrospective review was performed of all arteriovenous fistula (AVF) created in a HD population aged 0 to 18 years at a single institution from 2007 to 2019. Data abstracted included age, weight, etiology of renal failure, time on dialysis, central venous catheter history and transplantation history. Data were analyzed to determine the primary and secondary patency.

Results: During the study period, 19 AVFs were performed in 16 patients, of whom 9 patients (56.3\%) were male. Mean patient age was 12.3 years (range 5-17 years), and mean weight was $38.3 \mathrm{~kg}$ (range 12-83kg). At the time of AVF creation, 9 patients were on dialysis and 7 patients had a central venous catheter (CVC), with a median length of CVC dependence of 10 months. Procedures performed included 4 radiocephalic fistulas, 11 brachiocephalic fistulas and 4 brachiobasilic. Five accesses failed to mature (26.3\%). Mean follow-up was 6 years. The 2 -year primary and secondary patency rates were $92.3 \%$ and $100 \%$, respectively. The 4-year primary and secondary patency rates were $76.9 \%$ and $100 \%$, respectively. No thrombosis was documented during follow-up. During the postoperative period, 10 patients $(62.5 \%)$ received a kidney transplant, in a mean time of 23 months.

Conclusions: AVFs demonstrate excellent long-term patency in pediatric HD patients. No significant complications were reported and no thrombosis occurred.

Key-words: Pediatric nephrology, hemodialysis, arteriovenous fistula.

(C) 2021 Portuguese Journal of Nephrology \& Hypertension. Published by Publicações Ciência \& Vida This is an open access article under the CC BY-NC-ND license (http://creativecommons.org/licenses/by-nc-nd/4.0/).

\section{INTRODUCTION}

According to the United States Renal Data System (USRDS), hemodialysis is the renal replacement therapy modality more frequently used and approximately $81.5 \%$ of incident pediatric patients have started HD with a central venous catheter (CVC) ${ }^{1}$. In Portugal, peritoneal dialysis (PD) has historically been the preferred dialysis method; however hemodialysis (HD) has been growing as the initial treatment modality in end-stage chronic kidney disease in children - Figure 1.

Choosing the best vascular access option for pediatric HD patients remains challenging. Despite the international guidelines for fistula first in the adult hemodialysis population, the pediatric population usually utilizes central venous catheters as the primary dialysis access. Permanent vascular access in the form of an arteriovenous fistula
(AVF) is the preferred form of vascular access for most pediatric patients on maintenance HD therapy ${ }^{2}$; however the technical aspects of the procedure that are unique to the pediatric population, the expectation of a short waiting time for kidney transplantation and the need to cannulate every other day (with the pain and fear associated with it) limit its use. Moreover, pediatric patients are likely to need multiple forms of vascular access during their lifetime, making vascular access decisions even more important.

Knowledge of the end-stage renal disease patient's history and vascular access patency are critical to make appropriate choices. Our Pediatric Nephrology Unit is responsible for all pediatric patients requiring chronic hemodialysis, from Northern Portugal. Our objective was to analyze the long-term outcomes of pediatric arteriovenous fistulas in our institution. 


\section{Figure 1}

Prevalence of peritoneal dialysis (PO) and hemodialysis (HD) per year in portuguese pediatric population.

$\begin{array}{lllllllllll}35 & 38 & 35 & 35 & 34 & 25 & 33 & 28 & 24 & 23 & n\end{array}$

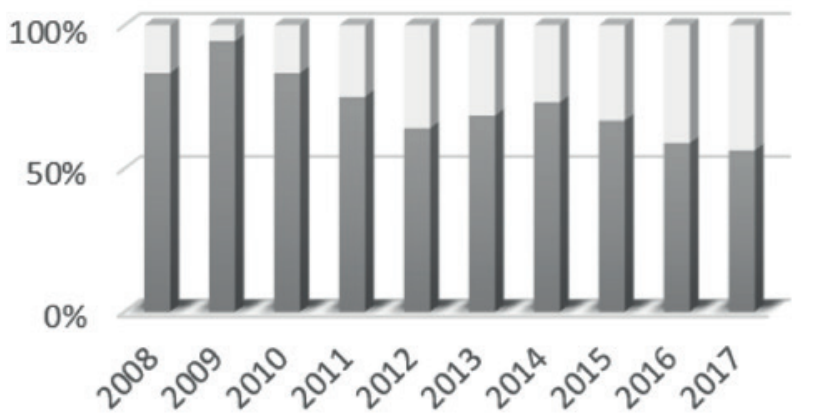

PD $\quad \mathrm{HD}$

Data from Portuguese Stage 5 Chronic Kidney Disease Prevalence 2017. Available at: https://www. spp.pt/UserFiles/file/Seccao_Nefrologia/registoIRCT\%202017.pdf Accessed Dctober 26, 2019.

\section{METHODS}

Study design: A retrospective review was performed of all AVFs created in a HD population aged 0 to 18 years at Centro Hospitalar Universitário do Porto from 2007 to 2019.

Data Collection and Description: All data were obtained from electronic patient records and surgical notes. Several variables were recorded: demographics (age, gender and weight), time and type of dialysis (PD or HD) and transplantation history. Clinical data and HD records were used to evaluate the follow-up.

Technique: The creation of each fistula was performed by vascular surgeons, and the type of access created was surgeon-dependent. Ultrasound vein mapping was performed on all patients and evaluated by each surgeon for suitability of vein size, and in general, the minimum size cutoff was $2.0 \mathrm{~mm}$ for both artery and veins. Papaverine was used at surgeon discretion. Procedures were performed with patient under general anesthesia. All AVFs were performed with a standard end-to-side anastomosis with a continuous running monofilament suture. Basilic vein transpositions were performed in a two-stage procedure, using the surgical technique described elsewhere ${ }^{3}$.

Outcomes: Access patency was defined and calculated according to published criteria 4 . Primary patency was defined as the time from AVF creation to any intervention performed to maintain patency or abandonment of the fistula for any reason without intervention. Secondary patency was defined as the patency of the AVF from creation to abandonment for any reason (including thrombosis, death, transfer to adult unit, transplantation and end of study period).

Statistical analysis: Summary results are reported as mean \pm standard deviation for continuous variables and as frequency (percent) for categoric variables.

\section{RESULTS}

During the study period, 19 AVFs were performed in 16 patients, of whom 9 patients (56.3\%) were male. Mean patient age was $12.1 \pm 3.4$ years (range, $5-17$ years), and mean weight was $38.3 \pm 18.1 \mathrm{~kg}$ (range, $12-83.1 \mathrm{~kg}$ ). At the time of AVF creation, 9 patients were already requiring renal replacement therapy; 7 patients were receiving $H D$ through a permanent CVC, with a median length of CVC dependence of 10 months; 7 patients had a peritoneal dialysis history; and 2 patients had already been submitted to kidney transplantation. All patient data and outcomes are given in Table 1. Etiology of renal failure was secondary to congenital anomalies of the kidney and urinary tract in 11 patients $(68.8 \%)$.

During this period, 12 other children underwent hemodialysis in our institution, using central venous catheter exclusively as vascular access. The reasons why no AVF creation was attempted were: hemodialysis for a short period of time ( $3.4 \pm 2.5$ months), either by kidney transplantation shortly after initiation of hemodialysis ( $n=7$ ) or Tenckoff catheter dysfunction and kidney transplantation after $(n=1)$; very young and small children $(n=2)$ of 2 and 5 years old; one death and one lost to follow up.

In the 16 patients submitted to AVF creation, the procedures performed were: 4 radiocephalic fistulas, 11 brachiocephalic fistulas and 4 brachiobasilic. Of the latter, three were subsequently submitted to basilic vein transposition - Figure 2 . Five accesses failed to mature (26.3\%). Mean follow-up was $70.4 \pm 38.3$ months (range, 7-146 months). The 2-year primary and secondary patency rates were $92.3 \%$ and $100 \%$, respectively. The 4 -year primary and secondary patency rates were $76.9 \%$ and $100 \%$, respectively. No thrombosis was documented during follow-up.

Functional maturation was never achieved in five fistulas (26.3\%): one radiocephalic, three brachiocephalic and one brachiobasilic AVF.

\section{Figure 2}

Basilic vein transposition with 3 short skip incisions.

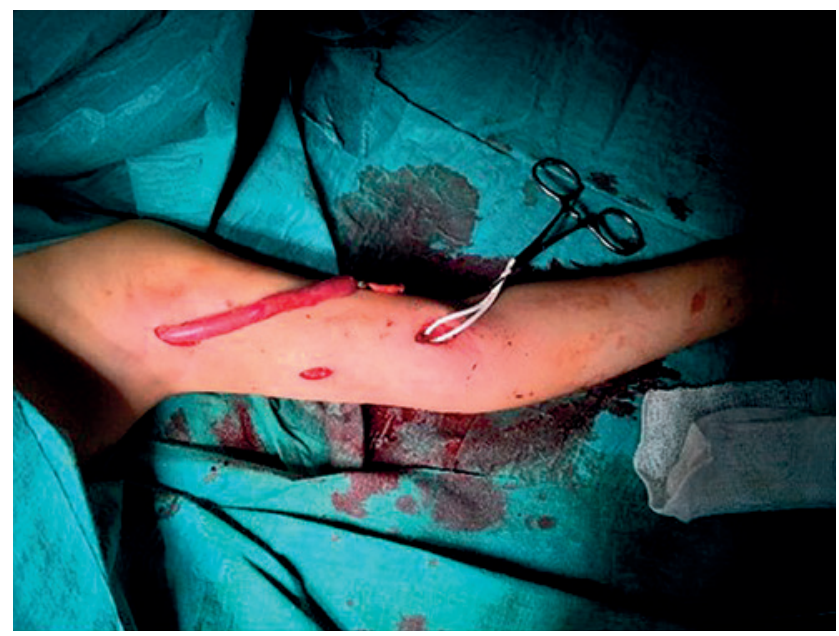




\section{Table 1}

Individual patient data and outcomes. All patients whose previous renal replacement therapy was hemodialysis (HD) had a central venous catheter. Secondary patency is the time of the AVF from creation to abandonment for any reason (includes death, transfer to adult unit, transplantation and end of study period).

\begin{tabular}{|c|c|c|c|c|c|c|c|c|}
\hline Patient & Age $(Y)$ & Gender & Weight (Kg) & $\begin{array}{c}\text { Previous } \\
\text { RRT }\end{array}$ & $\begin{array}{l}\text { AVF } \\
\text { Type }\end{array}$ & $\begin{array}{c}\text { Secondary } \\
\text { Patency (M) }\end{array}$ & Complications & Outcome \\
\hline 1 & 8 & Female & 12.2 & $H D$ & BVT & 44 & & $T x$ \\
\hline 2 & 10 & Male & 27.7 & $P D, T x, H D$ & $B C$ & 89 & Stenosis, HAIDI & $H D$ \\
\hline 3 & 15 & Female & 42.0 & HD & $\mathrm{RC}$ & 33 & Stenosis & $H D$ \\
\hline 4 & 8 & Female & 24.1 & $\mathrm{PD}, \mathrm{HD}$ & BC & 18 & & $T x$ \\
\hline 5 & 13 & Male & 43.0 & $\mathrm{Tx}$ & $\mathrm{RC}, \mathrm{BC}^{\mathrm{a}}$ & 52 & & $T x$ \\
\hline 6 & 9 & Female & 38.0 & $\mathrm{PD}, \mathrm{HD}$ & BVT & 54 & & Death \\
\hline 7 & 14 & Male & 40.6 & $P D, H D$ & $\mathrm{BC}, \mathrm{BC}^{\mathrm{a}}$ & 39 & & $\mathrm{Tx}$ \\
\hline 8 & 14 & Male & 45.8 & none & BVT & 42 & & Adult HD \\
\hline 9 & 14 & Male & 47.3 & none & $\mathrm{RC}$ & 48 & & Adult HD \\
\hline 10 & 16 & Male & 53.3 & PD & $\mathrm{RC}$ & 8 & HAIDI & $\mathrm{Tx}$ \\
\hline 11 & 5 & Male & 12.0 & $\mathrm{PD}, \mathrm{HD}$ & $B C$ & PF & & Death \\
\hline 12 & 17 & Male & 83.1 & none & $B C$ & 8 & & $T x$ \\
\hline 13 & 15 & Female & - & none & $\mathrm{BC}, \mathrm{BC}^{\mathrm{a}}$ & 81 & Aneurysm & $\mathrm{Tx}$ \\
\hline 14 & 11 & Female & 26.1 & none & BB & PF & & Tx \\
\hline 15 & 14 & Male & 41.0 & PD & $B C$ & 3 & & $T x$ \\
\hline 16 & 12 & Female & - & none & $B C$ & 48 & & $\mathrm{Tx}$ \\
\hline
\end{tabular}

${ }^{a}$ New ipsilateral brachiocephalic AVF creation after primary AVF failure.

AVF - arteriovenous fistula; BB - brachiobasilic; BC - brachiocephalic; BVT - basilic vein transposition; HAIDI - hemodialysis access induced distal ischemia; HD - hemodialysis; M - months; PD - peritoneal dialysis; PF - primary failure; PTA - percutaneous transluminal angioplasty; RC - radiocephalic; RRT - renal replacement therapy; Tx - kidney transplant; $Y$ - years; - missing data.

Two patients did not undergo new surgery: one died 7 months after the first surgery, and the other received a kidney transplant 9 months after. The other three patients (one radiocephalic and two brachiocephalic AVF) underwent new ipsilateral brachiocephalic AVF creation.

During the study period, 5 accesses (26.3\%) required revision. A radiocephalic AVF developed a stenosis in the mid-forearm radiocephalic vein, 6 months after construction, and underwent percutaneous angioplasty. A brachiocephalic AVF developed a postanastomotic stenosis 3.5 years after creation, and a new anastomosis was performed immediately after the post-anastomosis stenosis. A brachiocephalic AVF underwent revision for removal of postanastomotic venous aneurysm, 6.5 years after AVF creation. Hemodialysis access-induced ischemia occurred in two patients. The same patient submitted to brachiocephalic revision for stenosis presented 2 years later with cold hand and digital ulcers. This patient underwent an AVF banding, using intra-operative ultrasound control, with a flow reduction from $1000 \mathrm{~mL} / \mathrm{min}$ to $600 \mathrm{~mL} / \mathrm{min}$, with wound healing and resolution of pain afterwards. The second patient had a radiocephalic AVF and a well-functioning kidney transplant when he began ischemic complaints (coldness and pain at rest). In this patient, we decided on AVF ligation, performed 19 months after AVF creation. No thrombosis or AVF infection was registered. Two deaths were described during the follow-up, none related to the AVF access.

During the postoperative period, 8 patients $(57.1 \%)$ received a kidney transplant, in a mean time of 27 months ( 2 years).

\section{DISCUSSION}

Permanent access in the form of a fistula is consensually the preferred form of vascular access for most adult and pediatric patients on maintenance HD therapy ${ }^{2}$. The benefits of AVF creation include lower infection rate, lower thrombosis and stenosis rate, greater freedom with regard to activity ${ }^{5}$, and preservation of central veins.

There are technical aspects of the procedure that are unique to the pediatric population, with patients with smaller vessels and with more prominent intraoperative arterial spasm. When AVF was first attempted in children, a $50 \%$ immediate failure rate was report$\mathrm{ed}^{6}$. Our rate of failure before functional maturation was $26 \%(5 / 19)$, consistent with other major publications with failure rates of $5 \%$ to $33 \% 7$.

We report a 2-year primary and secondary patency of $92.3 \%$ and $100 \%$, and a 4 -year primary and secondary patency of $76.9 \%$ and $100 \%$. Bagolan et al ${ }^{8}$ divided patients by weight and described a 4 -year secondary patency of $70 \%$ in patients whose weight was more than $15 \mathrm{~kg}$ and $57 \%$ in patients whose weight was less than $15 \mathrm{~kg}$. More recently, Wartman et all ${ }^{9}$ reported a 2 -year primary and secondary patency of $83 \%$ and $92 \%$. Our success rate is within the described values. Systematic ultrasound mapping of arm vessels before surgical creation of dialysis access, the judicious choice of the best vessels, with minimum diameter of $2 \mathrm{~mm}$ in proximal and distal AVFs, and the use of papaverine to antagonize the arterial spasm may be essential for good results. Our rate of distal AVFs was $25 \%$, which is relatively 
low, either compared to adults or to other studies in children, and may be due to the criteria used and the existence of suitable vessels only in the elbow.

Pediatric patency numbers are greater than the patency values described in adults, with early failure rates of $2-46 \%$ and 1 -year secondary patency of $42-89 \%{ }^{10}$. In the adult population, age and diabetes mellitus negatively influence fistula maturation and increase the risk of AVF failure. In pediatric patients, the opposite seems true, and an association between increasing age and improved primary patency was demonstrated (hazard ratio, 0.88; $\mathrm{P} 0.02)^{9}$.

In our institution, a structured physical examination of AVFs is routinely performed by dialysis nurses and medical staff in every dialysis session with an urgent referral to a vascular access surgeon if AVF dysfunction or complications are detected on clinical examination. Possible complications of AVFs include stenosis, hematoma, thrombosis and distal ischemia. Bagolan et al. ${ }^{8}$ described a late complications rate of $26 \%$, due to thrombosis (79\%), hematoma (3\%) and stenosis (17\%). We present a very low rate of complications, with five reinterventions due to stenosis $(n=2)$, distal ischemia $(n=2)$ and post-anastomotic aneurysm $(n=1)$. No thrombosis was registered.

According to the USRSD data, since 2006, approximately $81.5 \%$ of incident pediatric ESRD patients have started HD with a catheter (ranging from $77.7 \%$ to $82.9 \%$ ). The predominant catheter use was observed across all age groups of children and adolescents ${ }^{1}$. The high prevalence of HD through a CVC is of particular concern, given two main reasons: the high rates of catheter-related infection and central venous stenosis associated with long-term CVC use. Despite this, catheter history did not affect patency in our study, and there were no reports of central venous stenosis signs/ symptoms. The same results were described by Wartman et al. ${ }^{9}$, and they hypothesized that somehow veins in pediatric patients could tolerate CVCs with a lower incidence of resultant stenosis or could have a more robust compensatory system to accommodate the arterialized venous flow of an AVF with an ipsilateral central vein occlusion or stenosis. However, these explanations remain theoretical.

The expectation of a short waiting time for kidney transplantation also limits AVF creation. Due to the small denominator for children on dialysis, and the priority for allocating kidneys from deceased donors under the age of 35 years to pediatric patients, deceased donor transplant rates are highest in children. Most of our patients (62.5\%) received a renal transplant during the study period.

We acknowledge some limitations to this study: retrospective study design, with no standardized recording of data, small simple size and missing data. Neither the precise vessel size or the AVF flow rate information were recorded in our study, which could be important to understand the primary failure rate and the (absence of) complications, for example the absence of central stenosis symptoms or thrombosis.

\section{CONCLUSION}

AVFs demonstrate excellent long-term patency in pediatric HD patients. No significant complications were reported and no thrombosis occurred. The good long-term results are due to the rigor and dedication of a multidisciplinary team involving pediatric nephrologists, experienced HD nurses and vascular surgeons.

Disclosure of potential conflicts of interest: none declared.

\section{References}

1. United States Renal Data System. USRDS 2018 Annual Data Report. Pediatric ESRD. Available at: http://www.usrds.org/adr.aspx. Accessed October 26, 2019.

2. Shroff R, Calder F, Bakkaloglu S, Nagler EV, Stuart S, Stronach L, et al. Vascular access in children requiring maintenance haemodialysis: a consensus document by the European Society for Paediatric Nephrology Dialysis Working Group. Nephrol Dial Transplant. 2019;34(10):1746-65.

3. Rego D, Nogueira C, Matos A, Almeida P, Queiros J, Silva F, et al. Two-Stage Basilic Vein Transposition: Second Stage Results. Ther Apher Dial 2018;22(1):73-8.

4. Sidawy AN, Gray R, Besarab A, Henry M, Ascher E, Silva M, Jr., et al. Recommended standards for reports dealing with arteriovenous hemodialysis accesses. J Vasc Surg. 2002;35(3):603-10.

5. Chand DH, Valentini RP, Kamil ES. Hemodialysis vascular access options in pediatrics: considerations for patients and practitioners. Pediatr Nephrol . 2009;24(6):1121-8.

6. Wander J, Moore E, Jonasson $\mathrm{O}$. Internal arteriovenous fistulae for dialysis in children. J Pediatr Surg. 1970;5:533-8.

7. Bourquelot $P$. Vascular access in children: the importance of microsurgery for creation of autologous arteriovenous fistulae. Eur J Vasc Endovasc Surg. 2006;32(6):696-700.

8. P Bagolan AS, G Ciprandi, S Picca, G Leozappa, A Nahom, A Trucchi, G Rizzoni, G Fabbrini. A tenyear experience of Brescia-Cimino arteriovenous fistula in children: technical evolution and refinements. J Vasc Surg. 1998;27:640-4.

9. Wartman SM, Rosen D, Woo K, Gradman WS, Weaver FA, Rowe V. Outcomes with arteriovenous fistulas in a pediatric population. J Vasc Surg. 2014;60(1):170-4.

10. Schmidli J, Widmer MK, Basile C, de Donato G, Gallieni M, Gibbons CP, et al. Editor's Choice Vascular Access: 2018 Clinical Practice Guidelines of the European Society for Vascular Surgery (ESVS). Eur J Vasc Endovasc Surg. 2018;55(6):757-818.

\section{ORCID}

Gabriela Teixeira (iD) 0000-0003-0077-7322

Paulo Almeida (iD) 0000-0002-6316-7751

A Norton de Matos (iD) 0000-0002-0544-0844

Maria Sameiro Faria (iD 0000-0002-8061-9289

Luís Loureiro (iD) 0000-0001-9740-018X

Duarte Rego (iD) 0000-0001-6529-1038

Inês Antunes iD 0000-0002-0526-4887

\section{Correspondence to:}

Gabriela Teixeira

Hospital de Santo António - Centro Hospitalar Universitário do Porto Largo do Prof. Abel Salazar, 4099-001 Porto, Portugal

E-mail: tgabrielateixeira@gmail.com 